\title{
Toxoplasma gondii RPL40 is a circulating antigen with immune protection effect
}

\author{
Junxin Xue ${ }^{1,2,3}$, Wei Jiang ${ }^{1}$, Jian Li $^{3}$, Wei Xiong ${ }^{3}$, Zhengan Tian ${ }^{3}$, Qiang Zhang ${ }^{3}$, Shuqing Li ${ }^{3}$, Chunfeng Liu ${ }^{3}$, \\ Kehe Huang ${ }^{2 *}$ and Quan Wang ${ }^{1 *}$
}

\author{
${ }^{1}$ Shanghai Veterinary Research Institute, Chinese Academy of Agricultural Science, Shanghai, P. R. China; \\ ${ }^{2}$ College of Veterinary Medicine, Nanjing Agricultural University, Nanjing, P. R. China; \\ ${ }^{3}$ Shanghai Customs, Shanghai, P. R. China
}

\begin{abstract}
Screening and identification of protective antigens are essential for the prevention of infections with Toxoplasma gondii (Nicolle et Manceaux, 1908). In our previous study, T. gondii ribosomal-ubiquitin protein L40 (TgRPL40) was identified as a circulating antigen. However, the function and protective value of TgRPL40 was unknown. In the current study, recombinant TgRPL40 was expressed in Escherichia coli BL21 and antibody was prepared. Western blotting analysis indicated that TgRPL40 was present in circulating antigens and excretory/secretary antigens (ESA). Immunofluorescence and immunoelectron microscopy analysis revealed that TgRPL40 protein is widely distributed in the tachyzoites. Immunisation with recombinant TgRPL40 prolonged the survival of mice infected with tachyzoites. Quantitative real-time polymerase chain reaction analysis showed that immunisation with recombinant TgRPL40 reduced the parasite burden in blood, liver, spleen and brain of mice infected with tachyzoites. These observations indicate that TgRPL40 is a circulating antigen and is an effector of immune protection against acute T. gondii infection.
\end{abstract}

Keywords: protein expression, subcellular localisation, protective effect

Toxoplasma gondii (Nicolle et Manceaux, 1908) is a major threat to human health and animal production. Infection with $T$. gondii is difficult to prevent and can cause serious illness. The parasite, which can infect all warm-blooded animals and proliferate in almost all nucleated cells, is the most widespread parasitic protist (Hunter and Sibley 2012). The majority of animals infected with T. gondii, including humans, typically exhibit no obvious clinical symptoms. However, serious and even life-threatening clinical symptoms can be present in organ transplant and immunosuppressed patients at the time of the first infection with T. gondii Robert-Gangneux and Darde (2012). Additionally, infection with $T$. gondii during early pregnancy can lead to abortion and fetal malformation (Hill and Dubey 2013, Mahmoudvand et al. 2015). Given the lack of effective drug treatments for $T$. gondii infection, the development of preventive strategies are of particular importance (Elmore et al. 2010, Zhou et al. 2016).

Immunisation with virulence factor-based vaccines is an effective strategy for the prevention of toxoplasmosis (Cong et al. 2011, Yin et al. 2015). Previous studies have shown that immunisation with $T$. gondii recombinant proteins, including cyclophilin, calcium-dependent protein kinase 1 , and actin, can stimulate the production of specific anti- bodies with immune protective effects (Huang et al. 2012, Yu et al. 2013, Chen et al. 2014). A recent study also indicated that circulating antigens (CAg) play indispensable roles in the pathological processes of $T$. gondii infection (Xue et al. 2016). Excretory/secretary antigens (ESA) are a general term for a class of proteins secreted by $T$. gondii in serum-free medium in vitro. CAg is the protein components of $T$. gondii in the blood of the host suffering acute toxoplasmosis. Previous studies have shown that most of the components of ESA and CAg are the same, such as surface antigens (SAG), micronemal proteins (MIC), dense granular proteins (GRA), and rhoptry proteins (ROP). (Zhou et al. 2005, Mattos et al. 2011, Meira et al. 2011).

Immunisation with ESA, which represent the majority of CAg, can effectively prolong the survival of mice infected with $T$. gondii Costa-Silva et al. (2008). Immunisation with recombinant ESA proteins, including SAG1, SAG2, ROP2, and GRA1, can also provide protective effects (Machado et al. 2010, Khosroshahi et al. 2012, Wu et al. 2012). However, antibodies targeting one or several virulence factors do not provide sufficient immune protective effects. In order to develop more effective protective strategies, more virulence factors with greater immunogenicity need to be further identified.

Address for correspondence: Q. Wang, Shanghai Veterinary Research Institute, Chinese Academy of Agricultural Science, No. 518, Ziyue Road, Minhang District, Shanghai, P. R. China. E-mail: wangquan@shvri.ac.cn;

K. Huang, College of Veterinary Medicine, Nanjing Agricultural University, No. 1, Weigang, Xuanwu District, Nanjing, P. R. China. E-mail: khhuang@, njau.edu.cn 
Ribosomal-ubiquitin protein L40 (RPL40) is fusion expressed with ubiquitin in eukaryotic cells. The fusion protein $\left(\mathrm{Ub}_{\mathrm{L} 40}\right)$ is a component of ribosome. $\mathrm{Ub}_{\mathrm{L} 40}$ can be decomposed into a ubiquitin monomer and a RPL40 molecule catalysed by a specific protease (Khatun et al. 2013). RPL40 regulates p53 expression in ribosomal protein - murine double minute $2-\mathrm{p} 53$ pathway (Zhou et al. 2019). In our previous study, we identified $T$. gondii ribosomal-ubiquitin protein L40 (TgRPL40) as a CAg component in the sera of dogs acutely infected with $T$. gondii Xue et al. (2016).

The results also suggested that TgRPL40 might be an essential virulence factor and may play a role in the pathogenesis of acute infection with $T$. gondii Xue et al. (2016). The aim of the current study was to investigate the localisation of TgRPL40 in tachyzoites. Considering that TgRPL40 can stimulate the body to produce antibodies, we evaluated the protective effect of immunising the virulence factor on $T$. gondii acute infection by immunising mice with recombinant TgRPL40.

\section{MATERIALS AND METHODS}

\section{Toxoplasma gondii, cells, and animals}

Toxoplasma gondii RH parasites and Vero cells were stored at the Parasite Laboratory of the Shanghai Veterinary Research Institute, Chinese Academy of Agricultural Science (Shanghai, China). Vero cells were propagated at $37^{\circ} \mathrm{C}$ and $5 \% \mathrm{CO}_{2}$ in Dulbecco's Modified Eagle's Medium supplemented with 10\% fetal bovine serum, $20 \mathrm{mg} / \mathrm{ml}$ penicillin and $20 \mathrm{mg} / \mathrm{ml}$ streptomycin. Toxoplasma gondii was grown and maintained in Vero cells.

Female ICR mice (body weight $\sim 25 \mathrm{~g}$ each) were purchased from the Shanghai Laboratory Animal Center, Chinese Academy of Science (Shanghai, China). All of the animals in the experiments were raised at the Animal Laboratory Center of the Shanghai Veterinary Research Institute in a sterile room and were fed sterilised food and water. The study was approved by the Animal Care and Use Committee of the Shanghai Veterinary Research Institute. Animals were handled in strict accordance with the animal protection law of the People's Republic of China (released on 18/09/2009) and the National Standards for Laboratory Animals in China (released on 01/05/2002).

\section{Bioinformatics analysis}

The secondary structure of TgRPL40 (GenBank: XP_002368400.1)was analysed using Protean software(DNAStar, Madison, WI, USA). The protein sequence of TgRPL40 was analysed using NCBI BLAST online tools (https://blast.ncbi.nlm. nih.gov/Blast.cgi). MegAlign software (DNAStar) was used for evolutionary tree analysis of the TgRPL40 gene sequence. Similarity comparisons with previously reported gene sequences were conducted using the online BLAST tool and DNAMAN 3.0 (Lynnon Biosoft, San Ramon, CA, USA). Multiple sequence alignment and phylogenetic analyses were conducted on the basis of observed similarities. Sequences were aligned with ClustalW2 (https://www.ebi.ac.uk/Tools/msa/clustalw2/). The phylogenetic tree was constructed using the neighbor-joining (NJ) method and plotted with MEGA 5.0 (http://www.megasoftware.net/).

\section{Design and synthesis of primers}

A pair of primers was designed by Primer 5.0 to amplify the rpl40 gene of T. gondii (GenBank: XM_002368359.1). BamHI and $\mathrm{Sal}$ I sites were introduced at the $5^{\prime}$ ends of the forward and reverse primers, respectively. The forward primer 5'-GTCGGATCCATGCAGATTTTCGTG-3' and the reverse primer 5'-ACGGTCGACTTAGTTCTTGGGCTTC-3' were used to amplify a 930 bp fragment.

\section{Parasite collection and RNA extraction}

Toxoplasma gondii $\mathrm{RH}$ was recovered from liquid nitrogen. When each well of the tissue culture flask was $90 \%$ confluent with Vero cells, $1 \times 10^{5}$ tachyzoites were inoculated into each well. Tachyzoites were collected by scraping and washing the flask with sterile saline three days post-inoculation. Tachyzoites were centrifuged at 1,000 rpm for $15 \mathrm{~min}$ and washed with phosphate buffered saline (PBS) three times prior to cell culture and RNA extraction. Total RNA was extracted by Trizol (Invitrogen, Carlsbad, CA, USA) and stored at $-70^{\circ} \mathrm{C}$ until use.

\section{PCR amplification and plasmid construction}

cDNA was obtained from $T$. gondii RH total RNA by reverse transcription using a Reverse Transcription Kit (Thermo Fisher Scientific, Waltham, MA, USA). Polymerase chain reaction (PCR) was performed using EX Taq DNA polymerase (Takara Bio Inc., Dalian, China) in a $50 \mu \mathrm{l}$ reaction mixture containing $2 \mu \mathrm{l}$ cDNA template. Reactions were performed for 30 cycles with denaturation at $95^{\circ} \mathrm{C}$ for $45 \mathrm{~s}$, annealing at $52^{\circ} \mathrm{C}$ for $50 \mathrm{~s}$, and extension at $72^{\circ} \mathrm{C}$ for $50 \mathrm{~s}$. Products were analysed by electrophoresis in $1 \%$ agarose gels with Goldview (SBS Genetech Co., Guangzhou, China).

The purified PCR products were inserted into the pMD19T vector. Plasmids were then transformed into Escherichia coli TOP10 competent cells (CWBIO Co., Beijing, China). Positive clones were screened on LB plates containing $100 \mu \mathrm{g} / \mathrm{ml} \mathrm{am-}$ picillin. Gene sequencing was performed on positive clones by Invitrogen. The plasmid containing the PCR product was named pMD19T-TgRPL40. The plasmid was extracted using the Plasmid Miniprep Kit (Axygen Scientific Inc., Corning, NY, USA). Plasmid pMD19T-TgRPL40 was digested with BamHI and Sal Iand inserted into the BamHI and Sal I sites of the pET-28a-c(+) expression vector. The sequence of the resulting plasmid was confirmed by dideoxy chain termination sequencing (Invitrogen).

\section{Induction, expression, and purification of recombinant pro- tein in Escherichia coli}

The expression plasmid was transformed into E. coli BL21 competent cells (CWBIO Co.). The transformed cells were grown in LB medium containing $70 \mu \mathrm{g} / \mathrm{ml}$ kanamycin at $37^{\circ} \mathrm{C}$. Protein expression was induced with $0.5 \mathrm{mM}$ isopropyl $\beta$-D-thiogalactopyranoside for $12 \mathrm{~h}$ at $20^{\circ} \mathrm{C}$. Pelleted cells were re-suspended and disrupted with a Sonicator (Scientz Biotechnology Co., Ningbo, China). The cell lysate was centrifuged for $30 \mathrm{~min}$ at $12,000 \times \mathrm{g}$ and the precipitate was suspended in $25 \mathrm{ml}$ binding buffer. After being filtred with a $0.22 \mu \mathrm{m}$ membrane, the recombinant protein in the supernatant was purified by Ni-NTA His-bind Resin (Novagen, Billerica, MA, USA). The purified His-TgRPL40 was dialysed in PBS at $4{ }^{\circ} \mathrm{C}$ for $12 \mathrm{~h}$ with stirring and then concentrated to $1 \mathrm{ml}$ using a $3 \mathrm{kDa}$ centrifugal filter (Merck, Darmstadt, Germany). The dialysed protein was stored at $-80^{\circ} \mathrm{C}$ until use. 


\section{Antibody production}

The concentration of the purified protein was measured using an RC DC Protein Assay kit (Bio-Rad, Hercules, CA, USA) after dialysis. Three mice were immunised with TgRPL40 protein absorbed to $100 \mu 1206$ adjuvant (Seppic, Paris, France) at a dose of $100 \mu \mathrm{g}$ per mouse. Immunisations were given at $0,2,4$, and 6 weeks. Control group mice were injected with the same volume of PBS containing 206 adjuvant. Immunisation procedures were assessed and antibody titres were measured by enzyme-linked immunosorbent assay (ELISA). Blood samples were collected 10 days after each injection. Serum was separated by centrifugation and stored at $-80^{\circ} \mathrm{C}$ until use.

ELISA was performed to measure antibody concentrations in microliter polystyrene plates (flat bottom, low binding; Corning, NY, USA). The purified tachyzoites were lysed by ultrasonic. The supernatant was separated by centrifugation and filtred by $0.45 \mu \mathrm{m}$ filter (Merck) to extract tachyzoite protein. A total of $500 \mathrm{ng}$ tachyzoite protein dissolved in $100 \mu \mathrm{l}$ coating buffer $(\mathrm{pH}$ 9.6) was added to each well. After incubating overnight at $4{ }^{\circ} \mathrm{C}$, each well was blocked with $200 \mu 1 \%$ gelatin at $37^{\circ} \mathrm{C}$ for $3 \mathrm{~h}$. Then, $100 \mu$ diluted serum $(1: 4000)$ in $5 \%$ bovine serum PBST (phosphate buffered saline $\mathrm{pH} 7.2$ containing $0.05 \% \mathrm{v} / \mathrm{v}$ Tween 20) was incubated at $37^{\circ} \mathrm{C}$ for $1.5 \mathrm{~h}$. After incubating with horseradish peroxidase-conjugated goat anti-mouse IgG (1 : 4000; Jackson Immuno Research Laboratories, Inc., West Grove, PA, USA), $100 \mu$ l substrate solution was added to each well. The reaction was stopped by adding $50 \mu 14 \mathrm{~N} \mathrm{H}_{2} \mathrm{SO}_{4}$. The absorbance was measured at $450 \mathrm{~nm}$ using an ELISA reader (Thermo). Each serum sample was assayed in duplicate.

\section{Western blotting}

Sera from three immunised mice were collected and combined 3 days after intraperitoneal injection with $T$. gondii RH strain to analyse whether TgRPL40 present in CAg. About $2 \mathrm{ml}$ of blood from each mouse was centrifuged at 2,000 rpm for 20 minutes to collect the serum. The sera were then filtred with $0.22 \mu \mathrm{m}$ filter (Merck). ESA were prepared according to the method described previously (Xue et al. 2016). The purified tachyzoites were suspended in $15 \mathrm{ml}$ serum-free DMEM medium and incubated in an incubator containing $5 \% \mathrm{CO}_{2}$ at $37^{\circ} \mathrm{C}$ for $2 \mathrm{~h}$. Then the medium was centrifuged at $4{ }^{\circ} \mathrm{C}$ for $15 \mathrm{~min}$ at $1,000 \mathrm{rpm}$. The supernatant was filtered by $0.45 \mu \mathrm{m}$ filter (Merck) after adding protease inhibitor (Merck, Darmstadt, Germany).

The filtered supernatant was concentrated to about $1 \mathrm{ml}$ through a protein concentrator with molecular weight of $3 \mathrm{kDa}$ (Merck). The concentration of ESA was detected by DC protein assay reagent package (Bio-Rad, Hercules, USA). Sodium dodecyl sulfate polyacrylamide gel electrophoresis (SDS-PAGE) was carried out with $100 \mu \mathrm{g}$ of each sample loaded into each well. Protein bands on gels were transferred onto nitrocellulose membranes $(0.45 \mu \mathrm{m}$; Amersham, Little Chalfont, UK) using a Transblot Semi-dry Transfer Cell (Bio-Rad). Mouse anti-TgRPL40 antiserum was used as the primary antibody $(1: 8000)$. Peroxidase-conjugated goat anti-mouse IgG (Jackson Immuno Research Laboratories, Inc.) was used as the secondary antibody. The membranes were visualised using a standard enhanced chemiluminescence system (Bio-Rad).

\section{Immunofluorescence assay}

Toxoplasma gondii-infected Vero cells grown on glass coverslips were fixed with $4 \%$ formaldehyde and permeabilised with $0.5 \%$ Triton-X100. The coverslips were blocked with PBS containing $1 \%$ bovine serum albumin (BSA) at $37^{\circ} \mathrm{C}$ for $30 \mathrm{~min}$. Mouse anti-TgRPL40 polyclonal antiserum (1:4000) was used as the primary antibody. Alexa Fluor 488-conjugated donkey anti-mouse IgG (Jackson Immuno Research Laboratories, Inc.) was used as the secondary antibody. Cells were stained with DAPI and images were acquired using Nikon Eclipse C1-Si confocal laser-scanning microscope (Nikon, Tokyo, Japan) with a $60 \times$ oil immersion lens. Three technical duplications were set for each sample.

\section{Immunoelectron microscopy}

For immunoelectron microscopy, extracellular tachyzoites were fixed with PBS containing $0.1 \%$ glutaraldehyde and embedded in LR white resin (Head Biotechnology Co., Beijing, China). Sections were treated with saturated sodium metaperiodate solution and incubated for $30 \mathrm{~min}$ in PBS containing 5\% skim milk and $0.01 \%$ Tween 20 (PBS-milk-Tween). Grids were then placed in PBST containing 5\% skim milk at $4{ }^{\circ} \mathrm{C}$ overnight. After washing with PBST containing 1\% BSA (PBS-BSA-Tween), the sections were incubated for $1 \mathrm{~h}$ with mouse anti-TgRPL40 polyclonal antibody (diluted 1 : 100 in PBS-milk-Tween). After washing with PBS-BSA-Tween, grids were incubated for $1 \mathrm{~h}$ with gold particle-labeled goat anti-mouse IgG $(1: 20$; 9-11 nm diameter; Cat No. SLBD7962V; Sigma-Aldrich Co., Santa Clara, CA, USA). The slides were then washed with PBS-BSA-Tween and distilled water. After staining with 50\% methanol containing 2\% uranyl acetate, the slides were observed using an electron microscope (Hitachi, Tokyo, Japan). Three technical duplications were set for each sample.

\section{Evaluation of the protective effects of recombinant TgRPL40 in mice}

To assess the immune protective effects of the recombinant TgRPL40 protein, mice were immunised with the protein at a dose of $100 \mu \mathrm{g}$ per mouse as described above. An equal volume of PBS containing 206 adjuvant was injected into mice in the control group. Fourteen days after the third immunisation, both control and immunised mice (10 mice per group) were injected intraperitoneally with $4 \times 10^{5}$ parasites. Blank group mice were injected with the same volume of sterile PBS. Cumulative mortality was plotted using a Kaplan-Meier survival plot and analysed using Microsoft Excel 2011 (Microsoft, Redmond, WA, USA).

\section{Analysis of tissue parasitism kinetics by quantitative real time PCR}

To further determine the protective effect of the recombinant TgRPL40 protein, immunised and control mice (10 mice per group) were infected intraperitoneally with $4 \times 10^{4} \mathrm{~T}$. gondii $\mathrm{RH}$ tachyzoites 14 days after the third immunisation. Two mice from each group were selected for collection of blood, liver, spleen, and brain on days 1, 3, 5, and 7 post-infection. To avoid sampling non-homogeneity in animals with low numbers of cysts, whole liver, spleen, or brain was ground in PBS. Blood, liver, spleen and brain tissue from the two mice from each group were combined. 


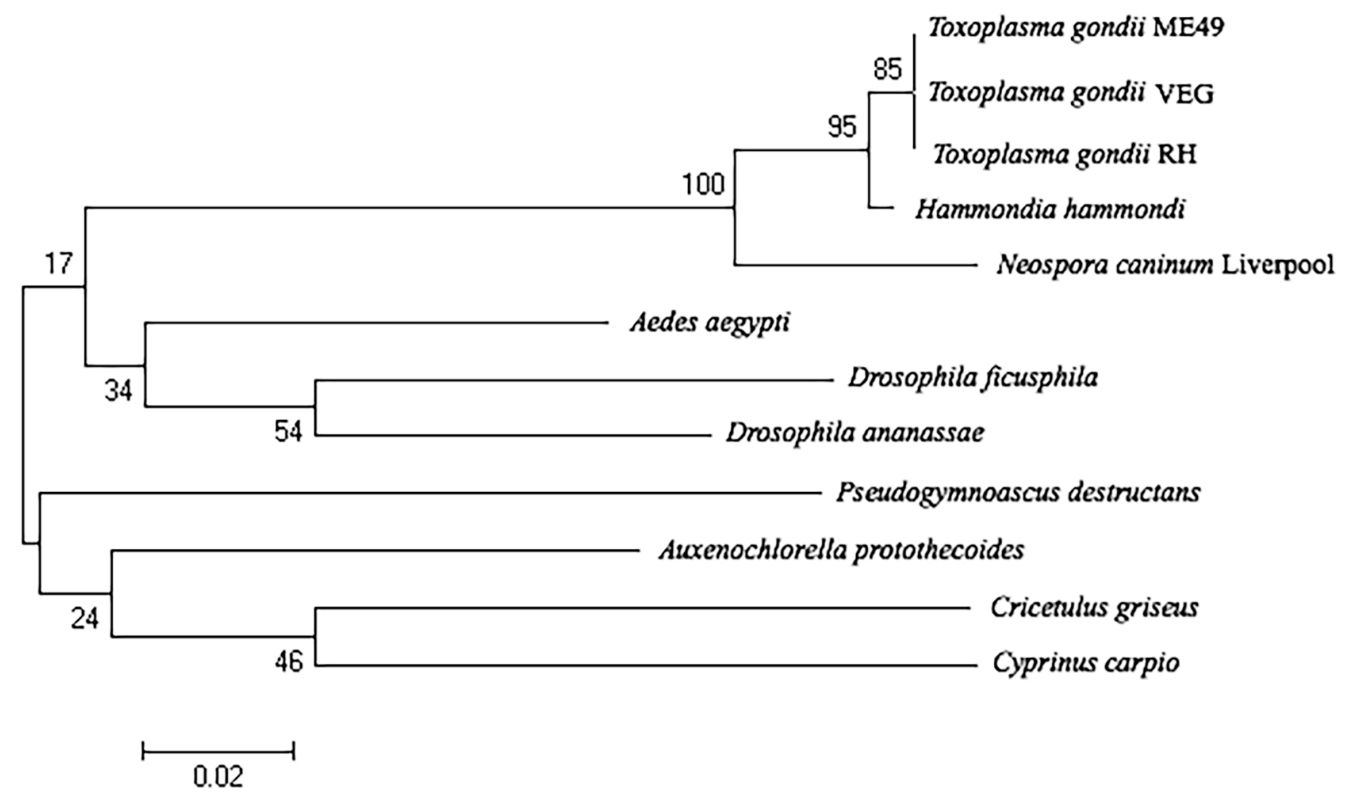

Fig. 1. Phylogenetic tree analysis of TgRPL40 sequence. Multiple sequence alignment and phylogenetic analyses were conducted on the basis of observed similarities. Sequences were aligned with ClustalW2. The phylogenetic tree was constructed using the neighbor-joining (NJ) method and plotted using MEGA 5.0.

Liver (30 mg), spleen (10 mg) and brain (30 mg) tissue deposition was collected by centrifugation. DNA was extracted using a TIANamp Genomic DNA kit (Tiangen Biotech Co., Beijing, China).

Tissue parasitism kinetics was estimated by quantitative real time PCR (qPCR) using an ABI 7500 PCR system (Applied Biosystems, Foster City, CA, USA) (Lin et al. 2012). Purified DNA was used in the reaction after diluting samples to equal concentrations of DNA. Each sample was assessed in triplicate. Six 10fold dilutions of $T$. gondii genomic DNA (0.1 ng to $1 \mathrm{fg}$ ) were used to calculate the standard curve. Each $25 \mu \mathrm{l}$ reaction $(0.4 \mu \mathrm{M}$ primers, $0.3 \mu \mathrm{M}$ probe and $1 \mu \mathrm{l}$ template) used the Probe qPCR Master Mix with uracil-DNA-glycosylase (Thermo Fisher Scientific). After $2 \mathrm{~min}$ at $50^{\circ} \mathrm{C}$ and an initial denaturation at $95^{\circ} \mathrm{C}$ for $10 \mathrm{~min}$, amplification consisted of 40 cycles of denaturation at $95^{\circ} \mathrm{C}$ for $15 \mathrm{~s}$, annealing at $52^{\circ} \mathrm{C}$ for $10 \mathrm{~s}$ and extension at $72^{\circ} \mathrm{C}$ for $15 \mathrm{~s}$. Parasite loads are presented as the numbers of tachyzoites in each $\mathrm{g}$ of tissue or per $\mathrm{ml}$ of blood.

\section{Statistical analysis}

Statistical analysis was performed using SPSS version 17.0 for Windows (SPSS Inc., Chicago, IL, USA). Data were analysed using one-way analysis of variance (ANOVA) followed by the least significant difference test. Data are expressed as mean \pm standard deviation. Differences were considered to be statistically significant at $\mathrm{P}<0.05$ or $\mathrm{P}<0.01$.

\section{RESULTS}

Phylogenetic tree analysis indicates that TgRPL40 is highly conserved in different species

NCBI online tools and DNAStar software were used to analyse the amino acid sequence of the TgRPL40 protein. BLAST results showed that the RPL40 protein is highly conserved in protists. The sequence of Toxoplasma gondii RH TgRPL40 is highly homologous to the protein sequences of RPL40 proteins from a variety of other $T$. gondii strains (identity $>95 \%$ ). Phylogenetic tree analysis showed that the sequence of the RPL40 protein of T. gondii RH, ME49, and VEG strains is highly homologous to the RPL40 protein of other protists, such as Hammondia hammondi Frenkel et Dubey, 1975 and Neospora caninum Dubey, Carpenter, Speer, Topper et Uggla, 1988 (Fig. 1) from Liverpool (identity $>95 \%$ ).

Compared with other species, differences in amino acid residues were found mainly at the $\mathrm{N}$ terminus. TgRPL40 contains 129 amino acid residues and amino acids 125-129 are -KKPKN. The amino acid sequences of the RPL40 protein of parasitic protists, such as H. hammon$d i$ and $N$. caninum (strain Liverpool), are the same as that of TgRPL40. In addition to parasitic protists, the RPL40 protein of Drosophila ficusphila Kikkawa et Peng, 1938 has the highest homology with TgRPL40. The RPL40 of D. ficusphila contains 128 amino acid residues and amino acids $125-128$ are -KKLK.

\section{Recombinant protein can be highly expressed by E. coli and is immunogenic}

Secondary structure analysis revealed high peptide epitope prediction scores for the peptide encoded by the 930 bp fragment. SDS-PAGE results showed that the recombinant TgRPL40 protein was expressed. The recombinant protein fused with a His-tag was $18.25 \mathrm{kDa}$ and could be purified by Ni-NTA resin (Fig. 2A). The antibody titres of the mice immunised with the recombinant protein were $1: 4000$ after the fourth immunisation. High titres indicated that the recombinant TgRPL40 protein was immunogenic (Fig. 2D). 

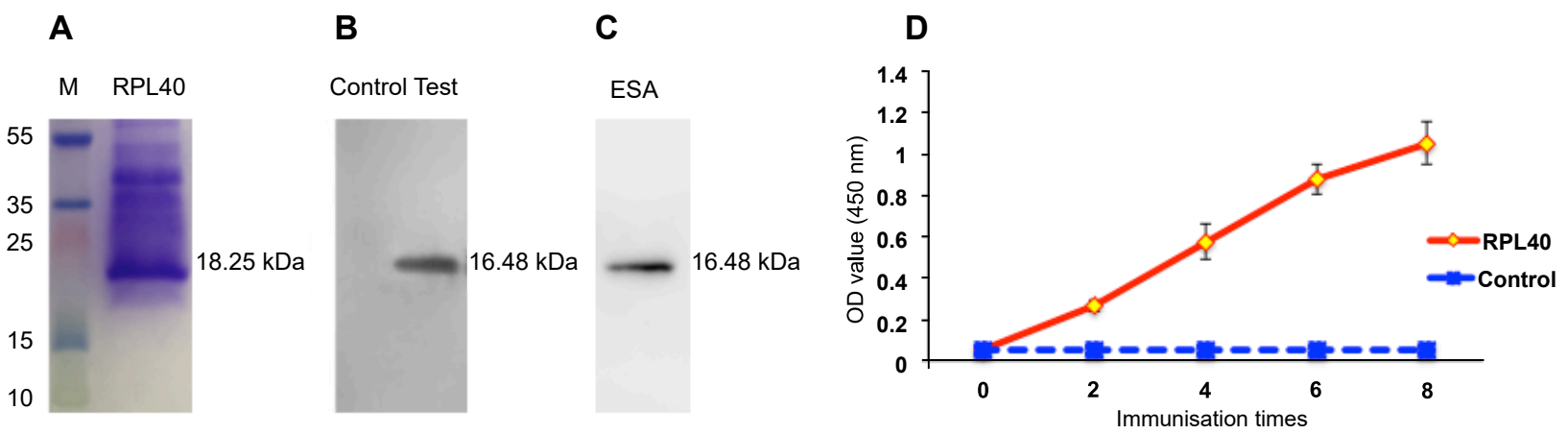

Fig. 2. Protein expression, Western blotting and antibody preparation. A - The recombinant TgRPL 40 protein was expressed in an Escherichia coli expression system and purified using Ni-NTA resin. The purified protein was analyzed by sodium dodecyl sulfate polyacrylamide gel electrophoresis (SDS-PAGE; 18\%) and the gel was stained with Coomassie brilliant blue-R250; B - TgRPL40 in the serum of mice acutely infected with Toxoplasma gondii (Nicolle et Manceaux, 1908) was identified with recombinant TgRPL40 antiserum. Control serum was obtained from three healthy mice; $\mathbf{C}-$ TgRPL40 in excretory/secretory antigens (ESA) was identified by recombinant TgRPL40 antiserum; D - Antibody titres were detected by enzyme-linked immunosorbent assay (ELISA). The interval between immunisations was two weeks. Sera were collected from the tails of mice approximately 2 weeks after each immunisation.

A

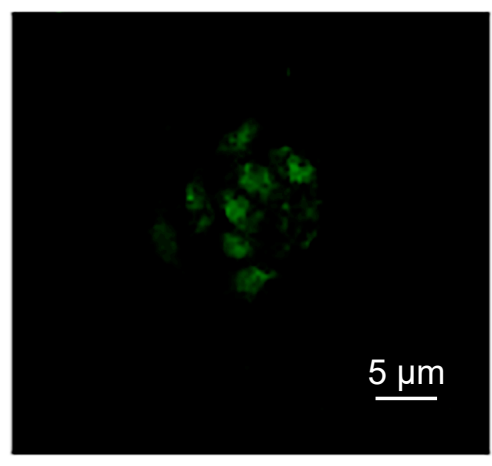

B

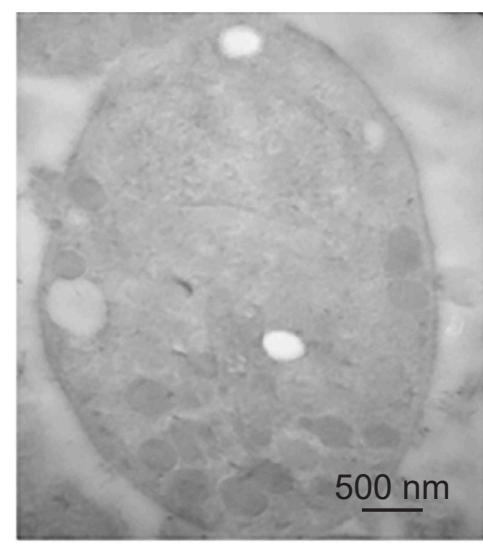

DAPI

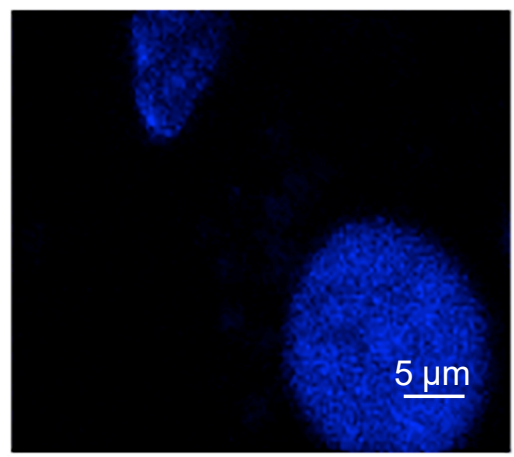

C

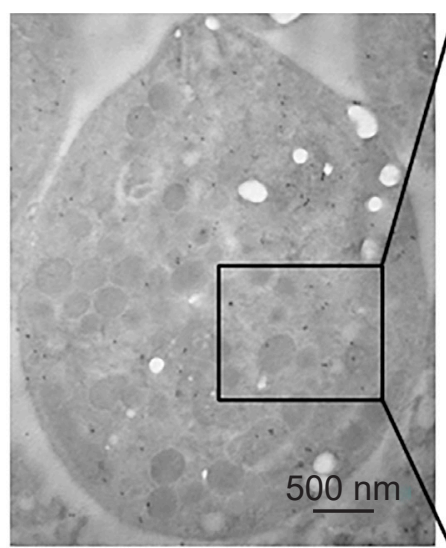

D
Merge
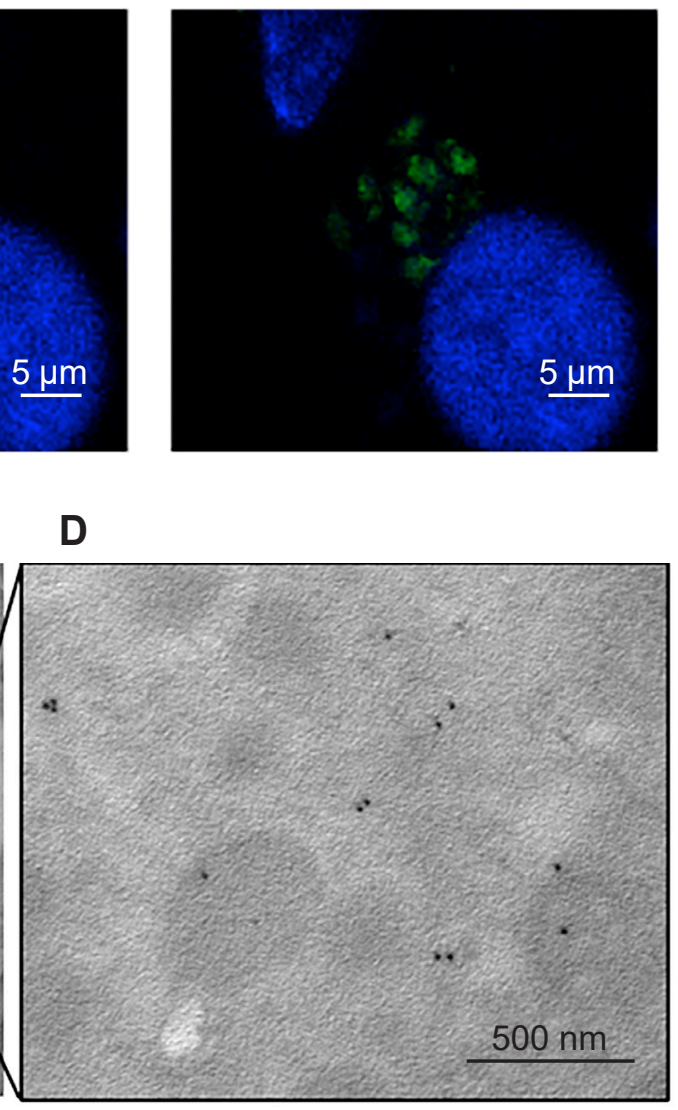

Fig. 3. Subcellular localisation using immunofluorescence and immunoelectron microscopy. A - subcellular localisation was performed using immunofluorescence; B - controls used in immunoelectron microscopy; C - Distribution of TgRPL40 in a longitudinal section of a tachyzoite; $\mathbf{D}$ - the enlargement of the local visual field in panel C.

\section{TgRPL40 is a component of CAg and ESA}

The present study showed that TgRPL40 could be detected by immunoblotting in sera of mice acutely infected with T. gondii (Fig. 2B). In addition, we identified TgRPL40 in the ESA (Fig. 2C). These results indicate that TgRPL40 is a CAg component, which is consistent with our previous proteomic study of acute infection with $T$. gondii infection Xue et al. (2016).

\section{TgRPL40 is widely distributed in the tachyzoites}

Indirect immunofluorescence assays showed that TgRPL40 was localised mainly in the cytoplasm of tachyzoites (Fig. 3A). To further investigate the potential function of 
A

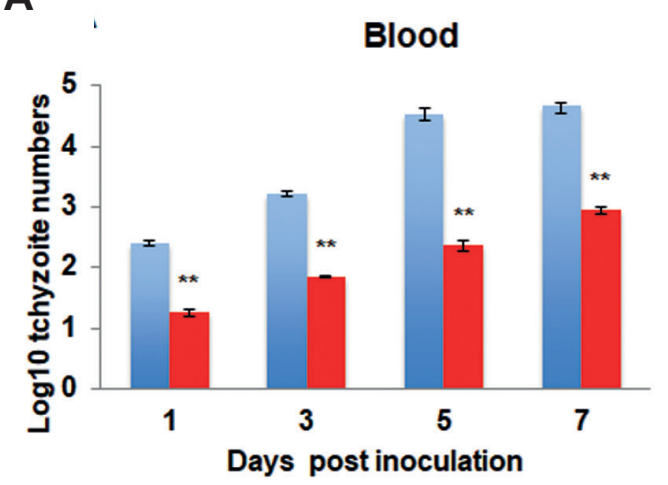

C

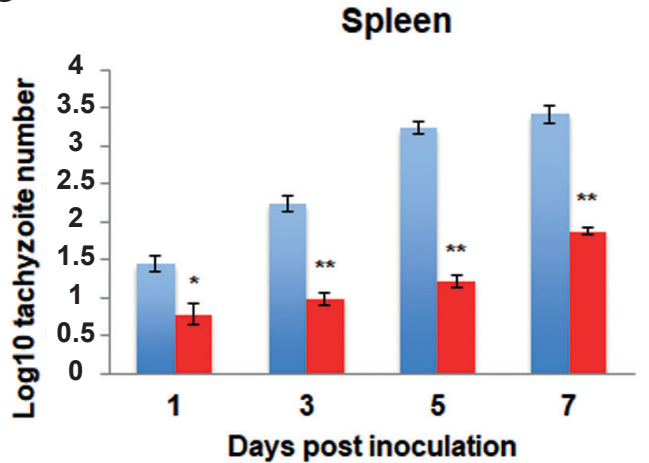

B

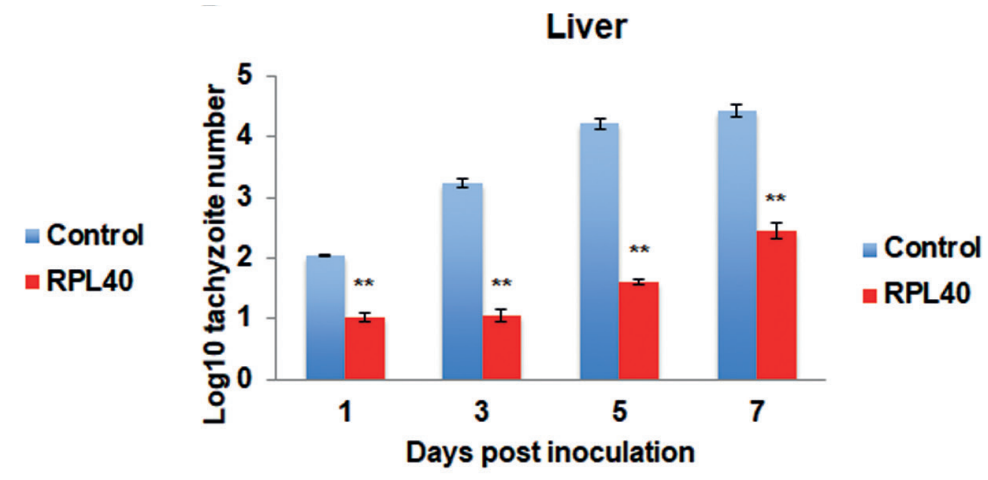

D

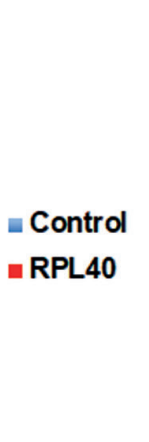

Brain

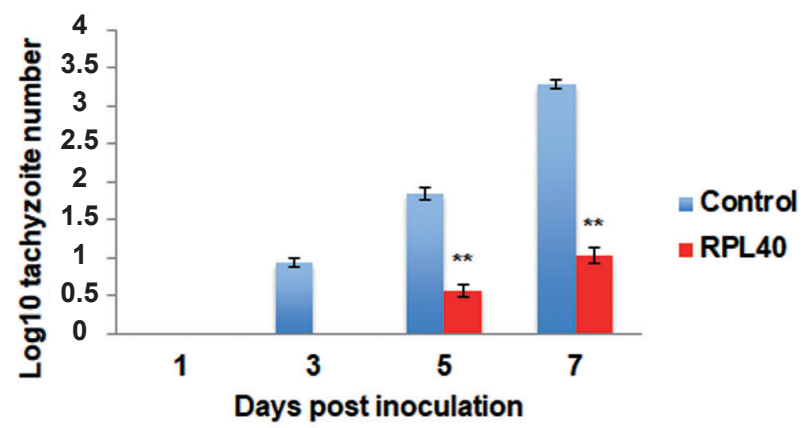

Fig. 4. Dynamic changes of Toxoplasma gondii (Nicolle et Manceaux, 1908) in blood, liver, spleen, and brain of mice. A-D - Ten mice in the protection group were immunised with recombinant TgRPL40 protein. Control group mice were immunised with the same volume of PBS. Each mouse was injected intraperitoneally with $4 \times 10^{4}$ parasites 14 days after the third immunisation. The numbers and dynamic changes of $T$. gondii in different tissues were detected by qPCR on days 1, 3, 5, and 7 post-infection. Values represent mean \pm standard deviation. Groups were compared using one-way analysis of variance (ANOVA) followed by a least significant difference test. Significant differences relative to controls are indicated by * $(\mathrm{P}<0.05)$ or ** $(\mathrm{P}<0.01)$.

TgRPL40, the intracellular location of the protein was determined using a more sensitive immunoelectron microscopy assay. The results of this assay confirmed that the TgRPL40 protein was widely distributed in the cytoplasm of tachyzoites. This distribution is consistent with the results of indirect immunofluorescence tests (Fig. 3B-D).

\section{Immunisation with recombinant TgRPL40 protein} reduces tachyzoite burdens in blood, liver, spleen, and brain

To monitor parasitemia and further assess the protective effects of recombinant TgRPL40 in mice, tissue parasitism kinetics were analysed by qPCR. The amplification efficiency for each test of $T$. gondii numbers in different tissues is shown in Supplementary Tab. 1. Tachyzoite numbers in the blood and livers of mice in the immunised group were significantly lower 7 days after infection (ANOVA test, $\mathrm{P}<0.01$ ) (Fig. 4A,B). Tachyzoite numbers were also significantly lower in spleen and brain of mice in the immunised group 7 days after infection (ANOVA test, $\mathrm{P}<0.05$ ) (Fig. 4C and 4D). Toxoplasma gondii became detectable by qPCR in brain tissue later than in the other tissues. These data suggest that immunisation with recombinant TgR-
PL40 exerts different degrees of inhibition on tachyzoites in blood, liver, spleen, and brain.

\section{Immunisation with recombinant TgRPL40 prolongs survival of mice infected with $T$. gondii}

To evaluate the protective effect of immunisation with recombinant TgRPL40 in mice, tachyzoites $\left(4 \times 10^{5}\right)$ were intraperitoneally injected into ICR mice ( $\sim 8$ weeks old) 14 days after the third immunisation. Mice immunised with recombinant TgRPL40 survived longer than mice that were immunised with PBS. However, mice immunised with the recombinant protein still eventually succumbed to the infection. All mice immunised with PBS died within 8 days, whereas mice immunised with recombinant TgRPL40 survived for 9 to 17 days (Fig. 5).

\section{DISCUSSION}

Our previous results indicated that TgRPL40 may play an essential role in the pathogenesis of acute infection with Toxoplasma gondii (see Xue et al. 2016). TgRPL40 is a fusion of ubiquitin and ribosomal protein L40. In most eukaryotic cells, ubiquitin is composed of 76 amino acid residues (Catic and Ploegh 2005). There are three precursor forms of ubiquitin including polyubiquitin and two N-ter- 


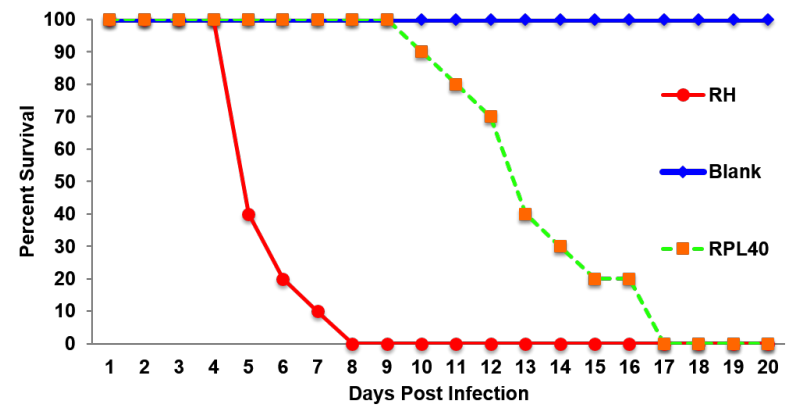

Fig. 5. Survival curves of mice infected with Toxoplasma gondii (Nicolle et Manceaux, 1908). Mice were immunised with recombinant TgRPL40. Control mice were administered phosphate buffered saline (PBS). Fourteen days after the third immunisation mice were injected intraperitoneally with $4 \times 10^{5}$ parasites. Blank group mice were injected with the same volume of PBS. Survival was monitored for 20 days.

minal ubiquitin moieties. The moieties UbL40 and UbS27 are fused with the ribosomal polypeptides L40 and S27, respectively. Cleavage of these precursor molecules by specific endopeptidases releases the ubiquitin monomers. The ubiquitin moiety then acts as a molecular chaperone to promote ribosome assembly before cleavage by L40 or S37 (Catic and Ploegh 2005).

Our results indicate that the pattern of TgRPL40 is similar to that of RPL40 in other species, which play roles in cytoplasm and some organelles (Wang et al. 2012, Fernandez-Pevida et al. 2012). The wide distribution of TgRPL40 in tachyzoites may be related to its physiological function. In other species, ubiquitin has been shown to have multiple roles during biological processes, including apoptosis, transcription and cell cycle, through covalent interactions with other proteins (Chi et al. 2016). First, it is well known that ubiquitylation is a biological signal of protein degradation. The isopeptide linkages between the glycine $(\mathrm{G}) 76$ and lysine (K) 48 or K29 of ubiquitin initiates enzyme-dependent degradation of ubiquitin binding proteins (Pickart 2000, 2001). Second, recent studies also indicate that ubiquitin plays an essential role in intracellular signal transduction (Su et al. 2013, Dores and Trejo 2019). Other biological processes include the regulation of ribosomal function (Spence et al. 2000), transcription (Bhat and Greer 2011), mitochondrial DNA inheritance (Lee et al. 2010) and DNA repair (Hedglin and Benkovic 2015).

Ribosomal proteins are also structural components of the ribosome and play a role in protein translation and processing of genetic information (Han et al. 2012). Ribosomal proteins usually surround rRNA cores of ribosomal subunits. Although the amino acids encoding the peptides are based on RNA, ribosomal proteins may play roles in simplifying the protein translation process and other biological processes outside of the ribosome (Lee et al. 2013, Gamerdinger 2016). In this study, subcellular localisation indicated that the ribosomal TgRPL40 fusion protein has functions in the cytoplasm of tachyzoites.

The TgRPL40 antigen was identified in ESA of $T$. gondii by immunoblotting. TgRPL40 has also been identified as a CAg through proteomic analysis of serum collected during acute toxoplasmosis (Xue et al. 2016). Because signal peptide prediction analysis showed that there is no signal peptide in TgRPL40, we speculate that this protein may be secreted via exosomes or by other mechanisms. Although previous studies have focused on the functions of RPL40, the function of TgRPL40 in T. gondii infection and other biological processes was not sufficiently analysed. Expression of RPL40 in HEK293 cells is required for the cells to transition from an adherent to a suspension phenotype after heat shock (Trakman et al. 2016).

RPL40 plays a role in the formation of vesicular stomatitis virus $80 \mathrm{~S}$ mRNA through a cis-regulatory element (Lee et al. 2013). In the current study, we observed a high degree of homology with other parasitic protists, such as Hammondia hammondi and Neospora caninum, suggesting that TgRPL40 may have similar functions in counteracting host immune mechanisms. Similarly, human IgM and IgG antibodies have been shown to demonstrate high sensitivity and specificity for ribosomal ubiquitin proteins and the polyubiquitin of $T$. gondii Saadatnia et al. (2011). Why immune recombinant TgRPL40 protein can cause immune protection and the mechanism of its antibodies to play an immune protective role remain to be further studied.

Our study demonstrates that $T$. gondii ubiquitin can be expressed in the form of a fusion protein with ribosomal subunit L40 in tachyzoites. Immunisation with recombinant TgRPL40 can prolong the survival time of mice infected with $T$. gondii. We also demonstrated that recombinant TgRPL40 reduces the tachyzoite burden in blood, liver, spleen and brain of mice infected with $T$. gondii. These outcomes show that TgRPL40 is a potential candidate molecule for the prevention of $T$. gondii. Although immunisation with TgRPL40 alone was not able to prevent the death of mice infected with $T$. gondii, further study of the protective effects of TgRPL40 combined with other virulence factors will identify optimal combinations of virulence factors for the development of vaccines for clinical application.

Acknowledgments. This work was supported by National Key Research and Development Program of China (Grant No. 2016YFD0501100， 2016YFC1202000， 2016YFC1202004) and Shanghai Natural Science Foundation of China (Grant No. 17ZR1437300, 18ZR1413400). 


\section{REFERENCES}

Bhat K.P., Greer S.F. 2011: Proteolytic and non-proteolytic roles of ubiquitin and the ubiquitin proteasome system in transcriptional regulation. Biochim. Biophys. Acta. 1809: 150-155.

Catic A., Ploegh H.L. 2005: Ubiquitin-conserved protein or selfish gene. Trends Biochem. Sci. 30: 600-604.

Chen J., Li Z.Y., Huang S.Y., Petersen E., Song H.Q., Zhou D.H., ZHU X.Q. 2014: Protective efficacy of Toxoplasma gondi calcium-dependent protein kinase 1 (TgCDPK1) adjuvated with recombinant IL-15 and IL-21 against experimental toxoplasmosis in mice. BMC Infect. Dis. 14: 487.

Chi Q., Li F., Liu W., Xu Q., Hu J., Cheng Y., Jing X., Chenc L. 2016: Analysis of UB and L40 resistance related to deltamethrin in Drosophila kc cells. Gene 575: 399-406.

Cong H., Mui E.J., Witola W.H., Sidney J., Alexander J., Sette A., Maewal A., McLeod R. 2011: Towards an immunosense vaccine to prevent toxoplasmosis: protective Toxoplasma gondii epitopes restricted by HLA-A*0201. Vaccine 29: 754-762.

Costa-Silva T.A., Meira C.S., Ferreira I.M., Hiramoto R.M., Pereira-Chioccola V.L. 2008: Evaluation of immunization with tachyzoite excreted-secreted proteins in a novel susceptible mouse model (A/Sn) for Toxoplasma gondii. Exp. Parasitol. 120: 227-234.

Dores M.R., Trejo J. 2019: Endo-lysosomal sorting of G-protein-coupled receptors by ubiquitin: diverse pathways for G-protein-coupled receptor destruction and beyond. Traffic 20: 101109.

Elmore S.A., Jones J.L., Conrad P.A., Patton S., Lindsay D.S., Dubey J.P. 2010: Toxoplasma gondii: epidemiology, feline clinical aspects, and prevention. Trends Parasitol. 26: 190-196.

Fernandez-Pevida A., Rodriguez-Galan O., Diaz-Quintana A., Kressler D., De la Cruz J. 2012: Yeast ribosomal protein L40 assembles late into precursor $60 \mathrm{~S}$ ribosomes and is required for their cytoplasmic maturation. J. Biol. Chem. 287: 38390-38407.

Gamerdinger M. 2016: Protein quality control at the ribosome: focus on RAC, NAC and RQC. Essays Biochem. 60: 203-212.

Han X.J., Lee M.J., Yu G.R., Lee Z.W., Bae J.Y., Bae Y.C., KANG S.H., Kim D.G. 2012: Altered dynamics of ubiquitin hybrid proteins during tumor cell apoptosis. Cell Death Dis. 3: e255.

Hedglin M., Benkovic S.J. 2015: Regulation of Rad6/Rad18 activity during DNA damage tolerance. Annu. Rev. Biophys. 44 207-228.

Hill D.E., Dubey J.P. 2013: Toxoplasma gondii prevalence in farm animals in the United States. Int. J. Parasitol. 43: 107-113.

Huang X., Li J., Zhang G., Gong P., Yang J., Zhang X. 2012 Toxoplasma gondii: protective immunity against toxoplasmosis with recombinant actin depolymerizing factor protein in BALB/c mice. Exp. Parasitol. 130: 218-222.

Hunter C.A., Sibley L.D. 2012: Modulation of innate immunity by Toxoplasma gondii virulence effectors. Nat. Rev. Microbiol. 10: 766-778

Khatun F., Kurata K., Chumattanakul V., Sugiyama M., Kaneko Y., Harashima S. 2013: Increased transcription of RPL40A and RPL40B is important for the improvement of RNA production in Saccharomyces cerevisiae. J. Biosci. Bioeng. 116 : 423-432.

Khosroshahi K.H., Ghaffarifar F., Sharifi Z., D'Souza S., Dalimi A., Hassan Z.M., Khoshzaban F. 2012: Comparing the effect of IL-12 genetic adjuvant and alum non-genetic adjuvant on the efficiency of the cocktail DNA vaccine containing plasmids encoding SAG-1 and ROP-2 of Toxoplasma gondii. Parasitol. Res. 111: 403-411.
Lee A.S.Y., Burdeinick-Kerr R., Whelan S.P.J. 2013: A ribosome-specialized translation initiation pathway is required for cap-dependent translation of vesicular stomatitis virus mRNAs. Proc. Natl. Acad. Sci. U. S. A. 110: 324-329.

Lee J.Y., Nagano Y., Taylor J.P., Lim K.L., YaO T.P. 2010 Disease-causing mutations in parkin impair mitochondrial ubiquitination, aggregation, and hdac6-dependent mitophagy. J. Cell Biol. 189: 671-679.

Lin Z.B., Zhang Y.L., Zhang H.S., Zhou Y.Z., CaO J., Zhou J.L. 2012: Comparison of loop-mediated isothermal amplification (LAMP) and real-time PCR method targeting a 529-bp repeat element for diagnosis of toxoplasmosis. Vet. Parasitol. 185: 296-300.

Machado A.V., Caetano B.C., Barbosa R.P., Salgado A.P., Rabelo R.H., Garcia C.C., Bruna-Romero O., Escriou N., Gazzinelli R.T. 2010: Prime and boost immunization with influenza and adenovirus encoding the Toxoplasma gondii surface antigen 2 (SAG2) induces strong protective immunity. Vaccine 28: $3247-3256$

Mahmoudvand H., Dezaki E., Soleimani S., Baneshi M., Kheirandish F., Ezatpour B., Zia-Ali N. 2015: Seroprevalence and risk factors of Toxoplasma gondii infection among healthy blood donors in south-east of Iran. Parasite. Immunol. 37: 362-367.

Mattos C.C., Meira C.S., Ferreira A.I., Frederico F.B., Hiramoto R.M., Almeida G.D.J., Mattos L.C., Pereira-ChiocCOLA V.L. 2011: Contribution of laboratory methods in diagnosing clinically suspected ocular toxoplasmosis in Brazilian patients. Diagn. Microbiol. Infect. Dis. 70: 362-366.

Meira C.S., Vidal J.E., Costa-Silva T.A., Frazatti-Gallina N., Pereira-Chioccola V.L. 2011: Immunodiagnosis in cerebrospinal fluid of cerebral toxoplasmosis and HIV-infected patients using Toxoplasma gondii excreted/secreted antigens. Diagn. Microbiol. Infect. Dis. 71: 279-285.

Pickart C.M. 2000: Ubiquitin in chains. Trends Biochem. Sci. 25: 544-548.

PiCKART C.M. 2001: Mechanisms underlying ubiquitination. Annu. Rev. Biochem. 70: 503-533.

Robert-Gangneux F., Darde M.L. 2012: Epidemiology of and diagnostic strategies for toxoplasmosis. Clin. Microbial. Rev. 25: 264-296.

Saadatnia G., Ghaffarifar F., Khalilpour A., Amerizadeh A., Rahmah N. 2011: A Toxoplasma gondii $10 \mathrm{kDa}$ in vitro excretory secretory antigen reactive with human $\operatorname{IgM}$ and $\operatorname{IgA}$ antibodies. Trop. Biomed. 28: 606-614.

Spence J., Gali R.R., Dittmar G., Sherman F., Karin M., FINLEY D. 2000: Cell cycle-regulated modification of the ribosome by a variant multiubiquitin chain. Cell 102: 67-76.

Su H., Chen M., Sands J.M., Chen G. 2013: Activation of the cAMP/PKA pathway induces UT-A1 urea transporter monoubiquitination and targets it for lysosomal degradation. Am. J. Physiol. Renal. Physiol. 305: F1775-F1782.

Trakman L., Hewson C., Burdach J., Morris K.V. 2016: RNA directed modulation of phenotypic plasticity in human cells. PLoS ONE 11: e0152424.

Wang Q., Chen L., Wang Y., Li W., He L., Jiang H. 2012: Expression characteristics of two ubiquitin/ribosomal fusion protein genes in the developing testis, accessory gonad and ovary of Chinese mitten crab, Eriocheir sinensis. Mol. Biol. Rep. 39: 6683-6692.

Wu X.N., Lin J., Lin X., Chen J., Chen Z.L., Lin J.Y. 2012 Multicomponent DNA vaccine-encoding Toxoplasma gondi GRA1 and SAG1 primes: anti-Toxoplasma immune response in mice. Parasitol. Res. 111: 2001-2009. 
Xue J., Jiang W., Chen Y., Liu Y., Zhang H., Xiao Y., Qiao Y., Huang K., Wang Q. 2016: Twenty-six circulating antigens and two novel diagnostic candidate molecules identified in the serum of canines with experimental acute toxoplasmosis. Parasit. Vectors 9: 374.

Yin H., Zhao L., Wang T., Zhou H., He S., Cong H. 2015: A Toxoplasma gondii vaccine encoding multistage antigens in conjunction with ubiquitin confers protective immunity to BAL$\mathrm{B} / \mathrm{c}$ mice against parasite infection. Parasit. Vectors 8: 498.

Yu Q., Huang X., Gong P., Zhang Q., Li J., Zhang G., Yang J., Li H., Wang N., Zhang X. 2013: Protective immunity induced by a recombinant BCG vaccine encoding the cyclophilin gene of Toxoplasma gondii. Vaccine 31: 6065-6071.
Zhou J., Lu G., He S. 2016: Analysis of structures and epitopes of a novel secreted protein MYR1 in Toxoplasma gondii. Folia Parasitol. 63: 028.

Zhou Q., Hou Z., Zuo S., Zhou X., Feng Y., Sun Y., Yuan X. 2019: LUCAT1 promotes colorectal cancer tumorigenesis by targeting the ribosomal protein L40-MDM2-p53 pathway through binding with UBA52. Cancer Sci. 110: 1194-1207.

Zhou X.W., Kafsack B.F.C., Cole R.N., Beckett P., Shen R.F., Carruthers V.B. 2005: The opportunistic pathogen Toxoplasma gondii deploys a diverse legion of invasion and survival proteins. J. Biol. Chem. 280: 34233-34244.

Cite this article as: Xue J., Jiang W., Li J., Xiong W., Tian Z., Zhang Q., Li S., Liu C., Huang K., Wang Q. 2019: Toxoplasma gondii RPL40 is a circulating antigen with immune protection effect. Folia Parasitol. 66: 013. 